\title{
Stochastic Asset Models for Actuarial Use in Ghana
}

\author{
Evans Tee ${ }^{1, *}$, Eric Dei Ofosu-hene ${ }^{2}$ \\ ${ }^{1}$ Department of Business Administration, Regentropfen College of Applied Sciences, Bolgatanga, Ghana \\ ${ }^{2}$ Department of Finance, University of Ghana Business School, University of Ghana, Legon, Ghana
}

Email address:

evans.tee@recas-ghana.com (E. Tee), eohene@ug.edu.gh (E. D. Ofosu-hene)

${ }^{*}$ Corresponding author

\section{To cite this article:}

Evans Tee, Eric Dei Ofosu-hene. Stochastic Asset Models for Actuarial Use in Ghana. International Journal of Statistical Distributions and Applications. Vol. 3, No. 4, 2017, pp. 129-139. doi: 10.11648/j.ijsd.20170304.20

Received: May 28, 2017; Accepted: June 12, 2017; Published: January 16, 2018

\begin{abstract}
The need for stochastic asset models has evolved from a common global standard for risk management in the Solvency II regime in Europe, IAIS Common Principles, Global ORSA standards NAIC, EIOPA, and OSFI. But the challenges in developing markets such as; lack of good quality data, inconsistent data coverage, market data not having long enough history, and lack of liquidity in certain parts of asset market have caused the absence of such models in Ghana. There have been a number of actuarial stochastic asset models designed for simulating future economic and investment conditions in several parts of the world. This study has discussed three of such models and determined which best fits the Ghanaian economic data. The data used for the empirical analysis in this study were taken from the Bank of Ghana database and the Ghana Stock Exchange. The study re-calibrated the models to derive the parameter set then compared the model results numerically after running 10000 simulations for 50 horizons. Investigations about the basic statistics of the simulated results for all the models are compared. The analysis revealed that all of the Ghanaian investment series used in the stochastic investment modeling are non-stationary in their mean, variance and auto-covariance. The study then found that the "Wilkie linear model" produced simulated values with similar characteristics to the historical data whiles the Whitten \& Thomas TAR model produced simulated values with minimal forecast error. The study therefore suggests that since the "Wilkie linear model" has a relatively better parsimony, ready economic interpretation and its ability to mimic some important features of the Ghanaian economic series it deserves the attention of the actuary seeking to model jointly the behavior of asset returns and economic variables that matter in economic capital determination of insurance and pension business in Ghana.
\end{abstract}

Keywords: Wilkie Linear Model, TAR Model, Stochastic, Asset, Simulations, Ghana

\section{Introduction}

Pension fund managers have been concerned with the issue of forming investment strategies that benefit their funds and more precisely how a pension fund should allocate its wealth among the different asset classes such that the risk in pension payments is minimized [1]. These risks mainly depend on macroeconomic factors. When actuaries are considering these financial risks, it is important to have a reasonable description of the investments as well as the liabilities extending far into the future. This, therefore, has led to the development and applications of models by actuaries and econometricians. Following [2] who derived a model of prices of ordinary shares in 1980 , actuaries have developed many stochastic asset models [3], [4], [5] etc.

Stochastic asset modeling involves projecting assets under a large number of equally probable randomly generated asset scenarios [6]. Its uses can be quite varied and include the development of probability distributions with regard to; statutory solvency, ability to meet contractual payments, and the examination of the resilience of management strategies among others. A stochastic investment model tries to project how investment returns on different assets such as equities or bonds vary over time. It is possible to use this to work out how investing in different assets could affect investments over time [7].

The benchmark to recent stochastic modeling is the [3] model. The Wilkie investment model ([3] and [8] are used widely to model investment returns by researchers who use 
stochastic simulation to investigate life office financial control [9]. Wilkie ([3]; [8]) developed linear stochastic asset models for United Kingdom data. Based on this model, a different model was built by [10] applying vector autoregression (VAR). [7] refined the price inflation component of the Wilkie model by performing a time series outlier analysis. Several researchers have developed stochastic investment models for several countries. Among these are: [11] for Switzerland, [12] for Canada, [13] for Finland, [4] for South Africa, [14] for the United States of America and [1] for Australia.

The need for stochastic asset models has evolved from a common global standard for risk management among the Solvency II requirements in Europe, International Association of Insurance Supervisors (IAIS) Common Principles, Global Own Risk and Solvency Assessment (ORSA) standards, National Association of Insurance Commissioners (NAIC), European Insurance and Occupational Pensions Authority (EIOPA), and Office of the Superintendent of Financial Institutions (OSFI) (Lau, 2014). Stochastic models present to the actuarial profession a tool which can be used for simulations of possible futures extending far for many years [3]. It is, therefore, necessary for every economy especially a more volatile economy like Ghana to have models for investments and actuarial uses which is contingent on the past and present economic factors.

Studies in the area of stochastic models for actuarial use in Ghana is limited to the best of the researchers' knowledge as at the time of the study. There are no works on models that describe the stochastic behavior of the Ghanaian investment variables which are fundamental to the financial decisionmaking of institutions and individuals. Stochastic asset models are capable for medium and long term financial simulations which can be used in capital adequacy, resilience reserving and asset allocation activities as well as for the immunization of short-term interest rate risk, investment policy determination and the general quantification and management of risk pertaining to those assets and liabilities [15]. This research, therefore, test the forecast ability of three models to the Ghanaian financial series. The result will show a clear way of future modeling to the data by revealing whether the data is better fitted by a linear, $\mathrm{ARCH}$ or a Threshold model.

The development of stochastic investment models for actuarial and investment applications have become an important area of interest to actuaries [16]. The purpose of the stochastic asset model is to provide economic variables which are necessary for: projecting future pension amounts; calculating the reserves required to meet future liabilities; calculating the return on the portfolio of assets held given the investment strategy adopted [17]. Stochastic modeling provides a better and clear appreciation of the environment in which the actuarial responsibilities reside and this enables improved decision making by incorporating more formally this closer approximation to reality. Stochastic models have been developed to quantify certain aspects of risk, and the financial assumptions underlying these models have become clearer as they have been compared with the theories and models of financial economics [1].

This study deals with the topical issue that is challenging the financial services industry and most precisely the actuarial profession in many markets. The study is an attempt to investigate the stochastic asset models necessary for actuarial use in Ghana. The research is hoped that its findings will set the pace for discussions that will result in developing a comprehensive risk management framework for the insurance and pension industry in Ghana.

\section{Literature Review}

\subsection{Stochastic Asset Modelling}

Stochastic programming is defined by the Committee on Stochastic Programming in 2011 as a framework for optimizing problems that involve uncertainty. Models through such methods are able to allow for the revelation of information in a timely progressive manner through multiple decision stages such that each decision is adapted to the available information [18]. Several different methods for formulation exist for stochastic problems. In this research, my attention is on the stochastic investment models. The majority of the investments by life insurance or pension funds institutions involve fixed-income security investment where there is a considerable influence of inflation and interest rate sensitivities on their liabilities. This is the reason why almost all investment models have interest rate and price inflation at their bases.

Stochastic modeling takes useful data from the past and combines it with the present to model the future. As far as possible, the structure of a model should be consistent with validated or widely accepted economic and financial theory. The theories and the models rely on empirical data for justification. A statistical analysis of historical data always provides useful insights into the features of past experience that the model will need to capture. The model structure is to be consistent with historical data and the parameter estimation will usually be based on the historical data.

Stochastic investment models are not to explain past movements in the investment series, and are not used to perfectly predict returns on assets in any future period in order to exploit potential trading profits. However, they are used purportedly to illustrate probable investment scenarios which are observable at some point in the future. The models are generally used to show a wide variety of investment situations to which an insurer or a pension fund might be exposed and this stresses on the point that stochastic asset models are very useful [19].

\subsection{Stochastic Asset Models}

Stochastic asset models are useful for generating economic scenarios. Actuaries are interested in analyzing timedependent variables that are specific to the pricing, reserving, or dynamical analyzing of insurance products. As mentioned in the introduction, stochastic time series modeling has 
attracted considerable attention from actuaries around the world in recent years. Modeling in the insurance industry is actually pioneered by [20] who introduced the principle of protection against interest rate risk. [3] and [8] built linear time series asset models for United Kingdom investment data. Wilkie's model in 1986 assumes that price inflation is the core of developing the other variables. He used a firstorder autoregressive (AR (1)) model to simulate and predict inflation. Through a cascade modeling approach, [3] interacted inflation with other investment variables to build a comprehensive model. It includes such variables as; dividends, dividend yields, and interest rates.

Since the development of the [3] model, the Actuarial stochastic investment modeling literature has recorded some critics of the model. One of such critics was [21], who criticized the [3] model regarding its data resources and manipulation, model construction and calibration, and its parameter estimations. The model was also criticized because the model did not capture the heteroscedastic nature of the series. [8] was developed to address nonnormality of the inflation series which was then modeled as an AR-ARCH model in 1995. [10] proposes an alternative model based on vector autoregression (VAR) whiles [7] refined the price inflation component of the Wilkie model by performing a time series outlier analysis.

Many of the actuarial stochastic investment models have been developed using the [22] linear modeling techniques (eg. Wilkie models). There has been a considerable appreciation in recent years of statistical research in nonlinear modeling. In the economics time series literature, there have works such as the works of [23] and [24]. Nonlinear investment models have the benefit of being able to capture irregularities, jumps, and time irreversibility which are mostly associated with financial and economic time series [25]. Because of that, it is argued that such models are able to provide a much wider range of possible scenarios for the actuarial time series data than do linear models [5].

Since the introduction of the basic class of "self-exciting" Threshold Autoregressive (TAR) models (SETAR) by [26] and [27], many classes of nonlinear models that stem from the threshold autoregressive framework have been proposed. One of such models considered in this research is developed by [5] in the United Kingdom.

Another notable model in the literature is the Cairns' model, [28] fashioned a model which is defined in terms of stochastic differential equations with driving Brownian motions. [28] developed a stochastic model for the combined term structure of rates of interest on fixed-interest bonds, rates of interest on index-linked bonds and consumer price inflation. It provides a framework which provides a relatively simple means of ensuring that nominal interest rates always remain positive. It is an equilibrium model which is driven by a multifactor Ornstein-Uhlenbeck process. The model exploits the normality of the distribution of the driving factors to derive prices for zero-coupon bonds and interest rates. It produces a model for consumer price inflation in which the rate of price inflation is equal to the difference between nominal and real short-term rates of interest adjusted for an inflation risk premium (this was to reflect a market preference for index-linked assets) and then subject to a zero mean error.

The "TY" model developed by [29] is also a robust stochastic investment model that has a clear design and is easy to communicate. It is built on some of the concepts of the Wilkie model developed in 1986, with the TY Model showing basically different and distinctive approach to modeling equities. The model was designed using modern statistical techniques as well as a wider data set. Upgrading the standard actuarial valuation into a stochastic risk assessment is the way of the future, and the TY Model was built with this objective in mind. The TY Model has already been successfully used for UK pension fund applications. It is also valuable Actuaries advising life insurance companies, general insurance companies and other funds with long-term liabilities [29].

[30] developed an investment model applying modern financial technology that is capable of generating values of both real and nominal interest rates, inflation, equity returns, and dividend payouts. A two-factor model was used for both interest rates and inflation variables, a regime-switching model for equities, and a one-factor autoregressive dividend yield model.

\section{Methodology}

The models are explained below showing the formulae and considerations of the nature of the variables as modeled by the respective authors. The formulae define how each variable is simulated and each model requires certain parameters. All the models have been calibrated from past data and the authors have generally given the values of the parameters from their fitted model, but by fitting different economic data, it requires re-calibration to derive the parameter set that is useful to the study. In order to compare the models in a certain respect, this study used the same data set for the parameter estimation.

a. The Wilkie Model

The Wilkie's model is a cascade structure encompassing various investment series. In [3] and [8], the inflation series is assumed to be the driving force for the other investment series. The investment series are linked together through a vigorous study and analysis based on a mixture of statistical evidence and economic assumptions.

\section{Formulae}

\section{(i). The Price Inflation Model}

Inflation, as measured by the retail prices index $(\mathrm{Q})$ or consumer price index (CPI), is modeled by a first order auto regressive (AR (1)) process. Wilkie's AR (1) price inflation model is of the form:

$$
Q_{t}=\left(Q_{t-1}\right) * \exp \left(I_{t}\right)
$$

Where $I_{t}$ is the force of inflation over year (t-1) to $(\mathrm{t})$ and it is given as: 


$$
I_{t}=\ln \left(Q_{t}-Q_{t-1}\right)
$$

Hence:

$$
\begin{gathered}
I_{t}=Q M U+Q A *\left(\left(I_{t-1}\right)-Q \mathrm{MU}\right)+\mathrm{QSD} * Q Z_{t} \\
Q Z_{t} \sim i i d N(0,1)
\end{gathered}
$$

That is $Q Z_{t}$ is a series of independent, identically distributed unit normal variates, (the assumption is that they have zero mean and unit standard deviation). Where QMU, QA and QSD are parameters to be estimated.

This model is described as that, each year the force of inflation is equal to its mean rate (QMU), plus a percentage of last year's deviation from the mean (QA), plus a random innovation which has zero mean and a standard deviation of QSD [3]. The assumption is that inflation, being the factor of economic uncertainty, depends only on past values of itself. There is significant autocorrelation at lag 1 , which provides statistical justification for inclusion of the $\left(I_{t-1}\right)$ variable, and no other economically plausible autocorrelation or partial autocorrelation is significant at $95 \%$ [5]. The AR (1) model of the force of inflation is a statistically stationary series (i.e., in the long run, the mean and variance are constant).

(ii). Share Yields Model

Share yields are modeled as a function of the current inflation rate and the history of their past trends. The Wilkie's AR (1) model of the share dividend yield is given as:

$$
\begin{gathered}
\ln \left(Y_{t}\right)=Y W * I_{t}+\ln (Y M U)+Y N_{t} \\
Y N_{t}=Y A *\left(Y N_{t-1}\right)+Y S D * Y Z_{t} \\
Y Z_{t} \sim \operatorname{iid} N(0,1)
\end{gathered}
$$

That is, $Y Z_{t}$ is a series of independent, identically distributed unit normal variates, (the assumption is that they have zero mean and unit standard deviation). Where, YMU, YA, YW and YSD are parameters to be estimated.

This model uses logarithmically transformed dividend yield, $\ln \left(Y_{t}\right)$ as the response variable. [8] described the model as that, at any date the logarithm of the dividend yield is equal to its mean value (ln YMU), plus a percentage of its deviation a year ago (YA) from the mean, plus an additional influence from inflation (YW) times the force of inflation in the previous year, plus a random innovation which has zero mean and a standard deviation of YSD.

\section{(iii). The Dividends Model}

The model for share dividends, where $D_{t}$ is the value of a dividend index on ordinary shares at time $t$, is given as:

$$
\begin{gathered}
D_{t}=D_{t-1} * \exp \left(\left(D W * D_{t}\right)+\left(D X * I_{t}\right)+D M U\right. \\
+\left(D Y * Y E_{t-1}\right)+D S D * D Z_{t} \\
D M_{t}=\left(D D * I_{t}\right)+(1-D D) * D M_{t-1}
\end{gathered}
$$

Defining $K_{t}$ as the logarithm of the increase in the share dividends index from year $t-1$ to year $t$, the Wilkie's MA (1) dividend yield model can also be represented as:

$$
\begin{gathered}
K_{t}=D W * D M_{t}+\left(D X * I_{t}\right)+D M U+\left(D Y * Y E_{t-1}\right) \\
+\left(D B * D E_{t-1}\right)+D S D * D Z_{t}
\end{gathered}
$$

Where, $K_{t}=\ln \left(D_{t}\right)-\ln \left(D_{t-1}\right)$.

Hence, [8] and [3] modeled for $\mathrm{P}(\mathrm{t})$, the value of a price index of ordinary shares at time $t$ as:

$$
P_{t}=D_{t} / Y_{t}
$$

Or

$$
\begin{gathered}
\ln \left(P_{t}\right)=\ln \left(D_{t}\right)-\ln \left(Y_{t}\right) . \\
Y Z_{t} \sim \text { iid } N(0,1)
\end{gathered}
$$

That is, $D Z_{t}$ is a series of independent, identically distributed unit normal variates, (the assumption is that they have zero mean and unit standard deviation). Where, DMU, DB, DW, DX, DSD, DY and DD are parameters to be estimated.

[8] described the model in words as: "in each year the change in the logarithm of the dividend index is equal to a function of current and past values of inflation, plus a mean real dividend growth (which is taken as zero), plus an influence from last year's dividend yield innovation, plus an influence from last year's dividend innovation, plus a random innovation which has zero mean and a standard deviation (DSD)."

(iv). Long Term Interest Rate

The long-term interest rate model is for the Consols yield $C_{t}$ The model is based on $C N_{t}$, which is $C_{t}$ adjusted the long memory effect of inflation rate. The Wilkie's AR (1) consols yield model is presented as:

$$
\begin{gathered}
C_{t}=C W * C M_{t}+C R_{t} \\
C M_{t}=C D * I_{t}+(1-C D) * C M_{t-1}
\end{gathered}
$$

This part is an exponentially weighted average of current and past price inflation, standing the expected future inflation over the life of the bond.

$$
\begin{gathered}
\ln \left(C R_{t}\right)=\ln (C M U)+C A *\left(\ln \left(C R_{t-1)}\right)-\ln (C M U)\right) \\
+\left(C Y * Y E_{t}\right)+\left(C S D * C Z_{t}\right)
\end{gathered}
$$

This is a zero-mean AR (1) process which is independent of price inflation and controls the long-term real interest rate.

$$
C Z_{t} \sim \operatorname{iid} N(0,1)
$$

That is, $C Z_{t}$ is a series of independent, identically distributed unit normal variates, (the assumption is that they have zero mean and unit standard deviation). Where, CMU, $\mathrm{CW}, \mathrm{CA}, \mathrm{CSD}$, and $\mathrm{CD}$ are parameters to be estimated.

The model is composed of two parts: an expected future inflation and a real yield [31]. The portion representing the inflation part is modeled as a weighted moving average whiles the real part is modeled is an AR (1) with a contribution from the dividend yield. The parameter, $C W=1$, which implies that the model takes into account, the "Fisher 
effect", in which the nominal yield on bonds reflects both expected inflation over the life of the bond and a 'real' rate of interest [31]. [8] defines the logarithm of the real interest component $\ln \left(C R_{t}\right)$ as a linear autoregressive order one or three AR (1) or AR (3) but preferred the AR (1) model.

(v). Short Term Interest Rate (Bank Rate)

Aside from the fundamental parts of the Wilkie model, this research considers one of the subsequent variables modeled by [8] Wilkie used bank rate or bank base rate series to model short-term interest rates. Short-term interest rates are clearly connected with long-term ones. Wilkie's approach was to model the difference between the logarithms of the difference of these series where $B_{t}$ is the value of bank rate at time $t$.

$$
\begin{gathered}
B_{t}=C_{t} * \exp \left(-B D_{t}\right) \\
B D_{t}=B M U+B A *\left(B D_{t-1}-B M U\right)+\left(B S D * B Z_{t}\right) \\
B Z_{t} \sim \text { iid } N(0,1)
\end{gathered}
$$

That is, $B Z_{t}$ is a series of independent, identically distributed unit normal variates, (the assumption is that they have zero mean and unit standard deviation). Where, BMU, $\mathrm{BA}$, and BSD, are parameters to be estimated.

\section{b. The Wilkie ARCH Model}

The initial model developed by [3] assumed that the residuals of the inflation model were normally distributed. In 1995, he re-examined his own model and observed that the residuals were much fatter tailed than a normal distribution. In Statistics and Econometrics, one of the ways to model these fat-tailed distributions is using an Autoregressive Conditional Heteroscedastic (ARCH) model [32] In an Autoregressive Conditional Heteroscedastic (ARCH) model, the variance of the innovation term is modeled as a separate process (rather than assumed to be constant) [10]. After the re-examination of the historical data, [8] proposed an ARCH model for the standard deviation of the inflation model.

The ARCH model was seen to describe the data better than the original model by [33] and was suggested that it should generally be used in applications of the model unless the $\mathrm{ARCH}$ effect is not significant for those particular applications. In this ARCH model the varying value of the standard deviation, QSD ( $t$ ), is made to depend on the previously observed value of the principal variable, $\mathrm{I}(\mathrm{t}-1)$, which itself is modeled by an autoregressive series. The suggested model (with a slight alteration in the notation) was:

$$
\begin{aligned}
& I_{t}=Q M U+Q A *\left(I_{t-1}\right)-Q M U+Q E_{t} \\
& Q E_{t}=\mathrm{QSD}_{t} * Q Z_{t} \\
& Q S D_{t}^{2}=Q S A^{2}+Q S B *\left(\left(I_{t-1}\right)-Q S C\right)^{2} \\
& I_{t}=\left\{\begin{array}{c}
Q M U 1+Q A 1 *\left(\left(I_{t-1}\right)-\mathrm{QMU} 1\right)+\mathrm{QSD} 1 * Q Z_{t} ; I_{t-1} \leq Q R \\
\mathrm{QMU} 2+\mathrm{QSD} 2 * Q Z_{t} ; I_{t-1}>Q R
\end{array}\right. \\
& Q Z_{t} \sim i i d N(0,1)
\end{aligned}
$$

$$
Q Z_{t} \sim i i d N(0,1)
$$

That is, $Q Z_{t}$ is a series of independent, identically distributed unit normal variates, (the assumption is that they have zero mean and unit standard deviation). Where, QMU, QA QSA QSB, and QSC, are parameters to be estimated. This implies that the variation depends on how far away last year's rate of inflation, $\left(I_{t-1}\right)$, was from some middle level, QSC (similar to the mean, QMU), but with the deviation squared, so that extreme values of inflation in either direction would increase the variance [19].

There have been some arguments that comparing the ARCH model to the initial autoregressive model shows that, the distribution of the force of price inflation $\left(I_{t}\right)$ exhibits fatter tails and a greater concentration around the long-term mean value. The ARCH variation was incorporated in only the price inflation model. Therefore, the remainder of the series follows the modeling as in the initial Wilkie model since [8] found no basses to re-model them as ARCH models. The ARCH model appears to give a better representation of inflation than the models assuming constant variance.

\section{c. The Whitten and Thomas Model}

The main underpinning belief for this model is that "the economy behaves differently in times of hyperinflation than it does in times of normal inflation levels" [5]. This belief is non-linear in nature and hence could not have been modeled linearly. After vigorous exploration of several alternatives, [5] adapted the Wilkie model (linear model) to incorporate their non-linearity assumption, rather than fundamentally changing the whole formulation.

[5] did not model the heteroscedastic nature of the price inflation using the ARCH model as in [8]) due to the challenges in estimating the model and to avoid tendency that it could give rise to troubling results from simulation. [5] employed the threshold modeling technique since threshold models are also capable of representing conditional variance, and moreover, exhibit short-term changes in mean. They proposed two regimes for each of the variables. The processes in each regime are similar to those defined by [3] and [8]. Following the same cascade structure above, the formulae for the models are given below:

\section{(i). The Price Inflation Model}

Inflation is assumed to be represented as a SETAR (selfexciting threshold autoregressive) model, with delay 1 , and a threshold that differentiates between normal and high inflation. They fitted many different threshold models. Due to the paucity of data partitioned into the upper regime, it was difficult to postulate any sort of autocorrelation structure in

The final suitable for threshold model for the price 
That is $Q Z_{t}$ is a series of independent, identically distributed unit normal variates, (the assumption is that they have zero mean and unit standard deviation). Where QMU1, QA1, QSD1, QMU2, and QSD2 are parameters to be estimated.

The model is described as that if the inflation in the previous year was below a certain threshold (QR), then the expected force of inflation $\left(I_{t}\right)$ is equal to its mean (QMU1), plus a percentage of last year's deviation from the mean (QA1) plus a random innovation which has zero mean and standard deviation QSD1. Conversely, if the inflation in the previous year was above the threshold, then the expected force of inflation presently is equal to its mean (QMU2), plus a random innovation which has zero mean and standard deviation QSD2. The model is able to control heteroscedasticity in a way because the expected variance of inflation when it is in its excited phase is greater than when it is in its quiescent phase [5].

(ii). The Dividend Model

Following [3] and [8], [5] also represented the share divided series as moving average of order one (MA (1)). Defining $K_{t}$ as in the Wilkie model as the logarithm of the increase in the share dividends index from year $t$ - 1 to $t$, this model is similar to the Wilkie model but with the introduction of a normal and a high inflation regimes. In economic sense, dividends do better in times of normal inflation, than in times of high inflation, therefore, the model employs the condition that $D M U 1>D M U 2$

The model for $K_{t}$ is of the form:

$$
K_{t}=\left\{\begin{array}{l}
D W * D M_{t}+\left(D X * I_{t}\right)+D M U 1+\left(D Y * Y E_{t-1}\right)+\left(D B * D E_{t-1}\right)+D S D * D Z_{t} ; I_{t-1} \leq Q R \\
D W * D M_{t}+\left(D X * I_{t}\right)+D M U 2+\left(D Y * Y E_{t-1}\right)+\left(D B * D E_{t-1}\right)+D S D * D Z_{t} ; I_{t-1}>Q R
\end{array}\right.
$$

Where,

$$
\begin{gathered}
D M_{t}=\left(D D * I_{t}\right)+(1-D D) * D M_{t-1} \\
D Z_{t} \sim \text { iid } N(0,1)
\end{gathered}
$$

That is $Q Z_{t}$ is a series of independent, identically distributed unit normal variates, (the assumption is that they have zero mean and unit standard deviation). Where, DMU1 DMU2, DB, DW, DX, DSD, DY and DD are parameters to be estimated.

(iii). The Share Yield Model

The share yield model is different to the Wilkie's $\ln \left(Y_{t}\right)$ in that they included a transfer effect from $\nabla C_{t}$ to $\ln \left(Y_{t}\right)$. $\ln \left(Y_{t}\right)$ was re-estimated as a TAR model, with extra parameters $Y Y 1$ and $Y Y 2$, to include this transfer, i.e.

$$
\ln \left(Y_{t}\right)=\left\{\begin{array}{l}
Y W * I_{t}+Y N_{t} ; I_{t-1} \leq Q R \\
Y W * I_{t}+Y N_{t} ; I_{t-1}>Q R
\end{array}\right.
$$

where,

$$
Y N_{t}=\left\{\begin{array}{c}
\ln (Y M U 1)+Y A 1 *\left(\left(Y N_{t-1}\right)-\ln (Y M U 1)\right)+Y Y 1 * \nabla \ln \left(C_{t}\right)+Y S D 1 * Y Z_{t} ; I_{t-1} \leq Q R \\
\ln (Y M U 2)+Y A 2 *\left(\left(Y N_{t-1}\right)-\ln (Y M U 2)\right)+Y Y 2 * \nabla \ln \left(C_{t}\right)+Y S D 2 * Y Z_{t} ; I_{t-1}>Q R \\
Y Z_{t} \sim \operatorname{iid} N(0,1)
\end{array}\right.
$$

That is, $Y Z_{t}$ is a series of independent, identically distributed unit normal variates, (the assumption is that they have zero mean and unit standard deviation). Where, YMU, YA, YW and YSD are parameters to be estimated.

(iv). Consol (Long-Term Interest Rate)

It was not easy to estimate the exponential smoothing parameter, $C D$, for each regime in $C_{t}$. Therefore, the parameter $C D$, was defined the same for each regime. It, therefore, follows that, like the Wilkie model, Whitten $\&$ Thomas' model gives a unit gain between inflation and interest rates.

The $C_{t}$ was then re-estimated as a TAR model, i.e.

$$
\begin{gathered}
C_{t}=C M_{t}+C R_{t} \\
\ln \left(C R_{t}\right)=\left\{\begin{array}{c}
\ln (C M U 1)+C A 1 *\left(\ln \left(C R_{t-1)}\right)-\ln (C M U 1)\right)+\left(C S D 1 * C Z_{t}\right) ; I_{t-1} \leq Q R \\
\ln (C M U 2)+C A 2 *\left(\ln \left(C R_{t-1)}\right)-\ln (C M U 2)\right)+\left(C S D 2 * C Z_{t}\right) ; I_{t-1}>Q R \\
C Z_{t} \sim \text { iid } N(0,1)
\end{array}\right.
\end{gathered}
$$


(v). Short-Term Interest Rate

The $B D_{t}$ in the Wilkie model was re-estimated as a TAR model, i.e.

$$
B D_{t}=\left\{\begin{array}{c}
B M U 1+B A 1 *\left(B D_{t-1}-B M U 1\right)+\left(B S D 1 * B Z_{t}\right) ; I_{t-1} \leq Q R \\
B M U 2+B A 2 *\left(B D_{t-1}-B M U\right)+\left(B S D 2 * B Z_{t}\right) ; I_{t-1}>Q R \\
B Z_{t} \sim \text { iid } N(0,1)
\end{array}\right.
$$

\section{Analysis and Findings}

\subsection{Moments}

Table 1 gives the descriptive statistics of the financial data typically used for the specification of the asset models considered in this work and the estimation of the models' parameters. These are compared to the model outputs in order to provide an insight into how better the models reflect the data from which the parameters were estimated.

Table 1. Descriptive Statistics of the Financial Data (1990-2013).

\begin{tabular}{llllllll}
\hline & MEANS & S. D & SKEW & KURT & MINI & MAX & MED \\
\hline$I_{t}$ & 0.1953 & 0.1079 & 1.579 & 2.325 & 0.08238 & 0.5353 & 0.1545 \\
$Y_{t}$ & 0.0401 & 0.0158 & -0.1032 & -1.6754 & 0.01600 & 0.0610 & 0.0430 \\
$C_{t}$ & 0.2206 & 0.0591 & -0.3756 & -1.1616 & 0.1130 & 0.3100 & 0.2270 \\
$B_{t}$ & 0.2743 & 0.1191 & 0.1808 & -1.0929 & 0.0960 & 0.4790 & 0.2710 \\
\hline
\end{tabular}

Source: Authors' Construct, 2014

The average historical force of inflation in Ghana $\left(I_{t}\right)$ is reported to be $19.53 \%$ which is very high compared to that of the UK used by [8] (4.2\%), also, both long-term interest rate $\left(C_{t}\right)$ and short-term interest rate $\left(B_{t}\right)$ historical averages have been higher in Ghana relative to the figures from the UK. The standard deviation and the skewness of the Ghanaian force of inflation series are also relatively higher than that of UK (0.05 and 0.97 respectively) but the kurtosis of the
Ghanaian series is relatively smaller than the UK force of inflation (4.29). Meanwhile, the historical average yield on ordinary shares in the UK $(5 \%)$ and other characteristics were higher than the yields in Ghana $\left(Y_{t}\right)$. This clearly shows the difference between the two Economies.

\subsection{Empirical Comparisons of the Models}

To better compare these models, simulated values of the economic variables were used. The comparison between the models is undertaken both in terms of comparing model outputs and making some general observations with regard to differences between the models.

\subsubsection{Basic Statistics of Simulated Results}

Tables 2 to 4 present the descriptive statistics for each of the economic series from each of the models based on 10000 simulations. The means and standard deviations together with the skewness and kurtosis are shown over various forecast horizons from year 1 to 50 years. It should be noted that with all the models, it is generally acceptable and easy to adjust the parameters to reflect the users own opinions for the mean and variance of each series. For the purposes of this research, the parameters were derived from the same data source with the same magnitude. The long-term mean variance, skewness and kurtosis for each series in the Wilkie model are quite similar to those of the ARCH model. The results from the Whitten \&

\begin{tabular}{|c|c|c|c|c|c|c|c|c|}
\hline & Period 1 & & & & Period & & & \\
\hline & Mean & St. Dev. & Skew. & Kurt. & Mean & St. Dev. & Skew. & Kurt. \\
\hline $\mathrm{I}(\mathrm{t})$ & 0.198 & 0.098 & 0.003 & 3.066 & 0.194 & 0.108 & 0.002 & 3.112 \\
\hline$Y(t)$ & 0.041 & 0.019 & 1.404 & 6.341 & 0.041 & 0.020 & 1.271 & 5.236 \\
\hline $\mathrm{K}(\mathrm{t})$ & 0.256 & 0.666 & 0.031 & 2.837 & 0.237 & 0.677 & 0.040 & 2.854 \\
\hline $\mathrm{P}(\mathrm{t})$ & 0.011 & 0.931 & 0.042 & 2.878 & 0.208 & 0.987 & 0.015 & 2.876 \\
\hline$C(t)$ & 0.459 & 0.111 & 0.353 & 3.227 & 0.440 & 0.134 & 0.379 & 3.960 \\
\hline \multirow{2}{*}{$B(t)$} & Period 20 & & & & Period & & & \\
\hline & Mean & St. Dev. & Skew. & Kurt. & Mean & St. Dev. & Skew. & Kurt. \\
\hline $\mathrm{I}(\mathrm{t})$ & 0.194 & 0.108 & 0.182 & 3.306 & 0.190 & 0.110 & -0.053 & 2.959 \\
\hline$Y(t)$ & 0.041 & 0.021 & 1.686 & 8.358 & 0.041 & 0.021 & 1.506 & 6.971 \\
\hline$K(t)$ & 0.302 & 0.675 & -0.01 & 2.828 & 0.298 & 0.674 & -0.113 & 2.849 \\
\hline$P(t)$ & 0.325 & 0.984 & 0.026 & 2.734 & 0.328 & 0.992 & -0.072 & 2.878 \\
\hline$C(t)$ & 0.430 & 0.140 & 0.493 & 3.637 & 0.430 & 0.133 & 0.287 & 3.179 \\
\hline
\end{tabular}
Thomas model vary slightly from the other two models.

Table 2. Results based on a sample of 10000 simulations of the Wilkie model.

Comparing the results to the actual data, it can be seen that 
models are quite similar to the original data. It has also revealed that the skewness and kurtosis from the simulations are higher than the original data. The kurtosis of the financial data for all the 4 investment series, except for the inflation series, are significantly lower than for the Wilkie model outputs. The higher kurtosis implied by the Wilkie model could have fewer consequences for applications where the tails of the investment distributions are more important.

Table 3. Results based on a sample of 10000 simulations of the ARCH model.

\begin{tabular}{|c|c|c|c|c|c|c|c|c|}
\hline & Period 1 & & & & Period 5 & & & \\
\hline & Mean & St. Dev. & Skew. & Kurt. & Mean & St. Dev. & Skew. & Kurt. \\
\hline $\mathrm{I}(\mathrm{t})$ & 0.196 & 0.100 & 0.039 & 2.971 & 0.189 & 0.110 & 0.027 & 2.803 \\
\hline$Y(t)$ & 0.040 & 0.020 & 1.758 & 8.893 & 0.040 & 0.021 & 1.581 & 7.658 \\
\hline $\mathrm{K}(\mathrm{t})$ & 0.274 & 0.674 & 0.000 & 2.856 & 0.268 & 0.676 & 0.058 & 2.876 \\
\hline $\mathrm{P}(\mathrm{t})$ & 0.057 & 0.960 & 0.005 & 2.836 & 0.286 & 1.024 & 0.049 & 2.987 \\
\hline$C(t)$ & 0.455 & 0.116 & 0.594 & 4.307 & 0.431 & 0.132 & 0.448 & 3.646 \\
\hline \multirow[t]{2}{*}{$B(t)$} & 0.346 & 0.094 & 0.714 & 4.130 & 0.332 & 0.111 & 0.598 & 3.823 \\
\hline & Mean & St. Dev. & Skew. & Kurt. & Mean & St. Dev. & Skew. & Kurt. \\
\hline $\mathrm{I}(\mathrm{t})$ & 0.197 & 0.109 & 0.009 & 2.861 & 0.195 & 0.107 & 0.008 & 2.893 \\
\hline$Y(t)$ & 0.041 & 0.020 & 1.398 & 5.883 & 0.042 & 0.022 & 1.555 & 6.827 \\
\hline $\mathrm{K}(\mathrm{t})$ & 0.265 & 0.688 & 0.077 & 2.808 & 0.236 & 0.689 & -0.077 & 2.866 \\
\hline $\mathrm{P}(\mathrm{t})$ & 0.266 & 0.986 & -0.051 & 3.124 & 0.239 & 1.008 & -0.164 & 3.020 \\
\hline$C(t)$ & 0.436 & 0.133 & 0.356 & 3.106 & 0.432 & 0.138 & 0.465 & 3.686 \\
\hline$B(t)$ & 0.333 & 0.110 & 0.613 & 3.896 & 0.332 & 0.115 & 0.513 & 3.320 \\
\hline
\end{tabular}

Comparing the results of the actual data, it can be seen that the inflation and dividend yield statistics from the Wilkie and $\mathrm{ARCH}$ models are quite similar to the original data. It has also revealed that the skewness and kurtosis from the simulations are higher than the original data.

Table 4. Results based on a sample of 10000 simulations of the Whitten \& Thomas model.

\begin{tabular}{|c|c|c|c|c|c|c|c|c|}
\hline & Period 1 & & & & Period 5 & & & \\
\hline & Mean & St. Dev. & Skew. & Kurt. & Mean & St. Dev. & Skew. & Kurt. \\
\hline $\mathrm{I}(\mathrm{t})$ & 0.146 & 0.041 & 0.039 & 2.971 & 0.146 & 0.047 & 1.092 & 8.077 \\
\hline$Y(t)$ & 0.141 & 1.333 & 26.501 & 753.9 & 1.282 & 18.86 & 30.106 & 931.08 \\
\hline$K(t)$ & 0.488 & 0.405 & -0.081 & 2.919 & 0.476 & 0.582 & 0.058 & 2.774 \\
\hline$P(t)$ & -0.181 & 1.995 & 0.033 & 2.907 & 0.587 & 4.563 & -0.213 & 3.229 \\
\hline $\mathrm{C}(\mathrm{t})$ & 0.366 & 0.075 & 0.609 & 4.087 & 0.351 & 0.079 & 0.708 & 3.855 \\
\hline \multirow[t]{2}{*}{$\mathrm{B}(\mathrm{t})$} & $\begin{array}{l}0.288 \\
\text { Period } 20\end{array}$ & 0.065 & 0.700 & 3.969 & $\begin{array}{l}0.278 \\
\text { Period } 40\end{array}$ & 0.068 & 0.631 & 3.561 \\
\hline & Mean & St. Dev. & Skew. & Kurt. & Mean & St. Dev. & Skew. & Kurt. \\
\hline $\mathrm{I}(\mathrm{t})$ & 0.153 & 0.059 & 2.388 & 14.08 & 0.152 & 0.06 & 2.702 & 16.835 \\
\hline$Y(t)$ & 0.453 & 2.214 & 15.039 & 277.9 & 0.513 & 2.55 & 9.208 & 98.64 \\
\hline$K(t)$ & 0.503 & 0.603 & 0.142 & 2.888 & 0.485 & 0.58 & -0.168 & 3.187 \\
\hline $\mathrm{P}(\mathrm{t})$ & 0.456 & 4.206 & -0.065 & 2.948 & 0.535 & 4.32 & -0.147 & 2.849 \\
\hline $\mathrm{C}(\mathrm{t})$ & 0.361 & 0.091 & 1.102 & 5.153 & 0.355 & 0.09 & 1.339 & 6.403 \\
\hline$B(t)$ & 0.282 & 0.073 & 0.798 & 4.103 & 0.278 & 0.08 & 1.001 & 4.998 \\
\hline
\end{tabular}

The financial data and the series in the WT model exhibit higher positive skewness than the series generated by the Wilkie model. The skewness and kurtosis are very large compared with the Wilkie model for the later forecast horizons. This implies that, for longer-term applications that mostly require more extreme fluctuations, the WT models could be used rather than the Wilkie models.

At this point, the long-term mean, variance and median for each series in the simulated values from the models have been compared to the original series. For the force of inflation and dividend yield series, the Wilkie models and the original data exhibit similar means, standard deviation and medians. The WT model expected values for the interest rates are quite closer to those of the actual data. It is noticeable that all the models were not able to capture the skewness and kurtosis of the original series. This is not surprising since the series do not exhibit the normal distribution assumed in the formulation of the models. To better utilize these models, other forms of distributions which can explain the fundamentals of the original series may be incorporated into the models.

\subsubsection{Forecast Error of the Models}

Empirically, the study examined the forecast errors of the models by comparing to the historical average of the series. The tables below report the various series with their root mean squared error (RMSE) over several horizons. Works such as [19] employed this technique. These measures are used as relative measure to compare forecasts for the same series across different models, the smaller the RMSE, the better the forecasting ability of that model accordingly. The measure of the deviation from the expected values (the means of the original data set) has been used for this purpose. Looking at the results presented in Table 1 and the simulated results, the average dividend yield was similar to the results 
from the Wilkie model whiles the averages of the long and short term interest rate as well as the force of inflation series were closer to the WT model. This is the reason why the WT model produced smaller values of the RMSE in the force of inflation, one year note and T-bill series compared to the other models.

Table 5. Forecast error: Force of Inflation.

\begin{tabular}{lllll}
\hline \multicolumn{5}{l}{ HORIZONS } \\
\hline MODELS & YEAR 1 & YEAR 5 & YEAR 10 & YEAR 50 \\
\hline WILKIE MODEL & 3.114295 & 3.370322 & 3.405916 & 3.310542 \\
ARCH MODEL & 3.232923 & 3.523303 & 3.473977 & 3.312842 \\
WHITTEN \& & 1.787686 & 1.988348 & 2.049345 & 1.916029 \\
THOMAS MODEL & & & & \\
\hline
\end{tabular}

Source: Authors' Construct, 2014

Table 6. Forecast error: Dividend Yield.

\begin{tabular}{lllll}
\hline \multicolumn{5}{l}{ HORIZONS } \\
\hline MODELS & YEAR 1 & YEAR 5 & YEAR 10 & YEAR 50 \\
\hline WILKIE MODEL & 0.6381973 & 0.6587442 & 0.6955593 & 0.7045382 \\
ARCH MODEL & 0.6397845 & 0.6876694 & 0.7751649 & 0.7818344 \\
WHITTEN \& & 42.259698 & 36.635634 & 2.336118 & 42.369329 \\
THOMAS MODEL & & & & \\
\hline
\end{tabular}

Source: Authors' Construct, 2014

Table 7. Forecast error: One Year Note.

\begin{tabular}{lllll}
\hline \multicolumn{5}{l}{ HORIZONS } \\
\hline MODELS & YEAR 1 & YEAR 5 & YEAR 10 & YEAR 50 \\
\hline WILKIE MODEL & 8.254858 & 8.137294 & 8.083348 & 7.864946 \\
ARCH MODEL & 8.266640 & 7.871529 & 8.085390 & 8.113343 \\
WHITTEN \& & 5.173571 & 4.821992 & 5.145882 & 5.144985 \\
THOMAS MODEL & & & & \\
\hline
\end{tabular}

Source: Authors' Construct, 2014

Table 8. Forecast error: 91 Day T-Bill.

\begin{tabular}{lllll}
\hline \multicolumn{5}{c}{ HORIZONS } \\
\hline MODELS & YEAR 1 & YEAR 5 & YEAR 10 & YEAR 50 \\
\hline WILKIE MODEL & 3.665743 & 3.981951 & 4.037623 & 3.825054 \\
ARCH MODEL & 3.773037 & 3.945236 & 4.124268 & 4.051031 \\
WHITTEN \& & 2.102078 & 2.148295 & 2.336118 & 2.305931 \\
THOMAS MODEL & & & & \\
\hline
\end{tabular}

Source: Authors' Construct, 2014

The results show that, consistently, the error values for the Whitten \& Thomas for the force of inflation, 91 day T-bill and the one-year bond yields are the least throughout the various horizons. The WILKIE model also shows consistent minimal forecast error among the three models in the dividend yield series. Since the models are designed for informing users about the shape of the asset return risks relative to central rates of return, rather than for predicting economic variables, the study does not place much emphasis on the fit of forecasts to out-of-sample data as a test of model validity to comparing the models. The study, therefore, used the results of the investigation of the median, standard deviation, skewness, and kurtosis statistics using the projection year simulation approaches as in the works of [17] and [5] together with the empirical cumulative distributions of the forecast series for the model comparison.

Looking at the simulated results, the Wilkie model and the ARCH model have produced similar values for the historical means and the standard deviations in the inflation and dividend yield series than the WT model. The Wilkie models were able to produce simulated results that have similar characteristics of the original data. However, the WT model forecasts the series with minimal error with the exception of the dividend yield series. The WT model produces relatively the closest short-term and long-term mean bond investment returns. It also exhibits one of the highest long-term standard deviations among the models. It is also one of the least volatile as it has one of the smallest standard deviations in the yield on long and short-term interest rates.

\section{Conclusion and Recommendations}

The results of the research suggest that the All Share Index (ASI) and the Consumer Price Index (CPI) from the Ghana investment annual data are not stationary also, the statistical analysis did not provide evidence that the interest rate and dividend yield were stationary too. Meanwhile, the log transformation and differencing of the CPI series resulted in a stationary series representing the annual force of inflation. All of the series used in stochastic investment modeling are non-stationary. That is their mean, variance and autocovariances may depend on time. Also, none of the series exhibited ARCH effect from the analysis. The evidence is clear that there is no enough evidence to accept the Gaussian distribution as a parametric approach in the modeling of financial returns for Ghana. This section provides some important considerations for future construction and usage of stochastic investment models in Ghana. It indicates the type of analysis that should be considered since this forms part of the foundation of an analysis of the series to be used in the models. These matters are fundamental to the construction of stochastic investment models.

The structures of the models are quite different but with same variables included and the frequency of the data used. The empirical comparison of the models has been done by comparing simulated values from the models to the original data. The results highlight that, the Wilkie model and the ARCH model have produced similar values for the historical means and the standard deviations in the inflation and dividend yield series than the WT model. The Wilkie models produce yield figures that have similar characteristics of the original data. However, the WT model forecasts the series with minimal error with the exception of the dividend yield series.

Looking at the characteristics of the Ghanaian financial series studied in this study, to build a more realistic stochastic asset model for actuary use in Ghana, it is significant to include time-varying parameters into developing such a model. Also, the results of any model cannot be accepted blindly since not all investment models are suitable for all applications. The Wilkie model is capable of producing values with similar features to the original data, 
however, the WT model can forecast most of the series with minimal error. However, based on the objective and empirical findings of this study, the Wilkie model is the "best" model for use in Ghana. The minimal error in the forecast of the WT model is as a result of the parameters and the initial values in the simulation process. Using the same initial parameters estimated from the lower regime or using the actual previous values for the simulation in the Wilkie model will produce a much better forecast of the Ghanaian financial series than the WT model.

The models presented are far from perfect. However, this research does believe that their relative parsimony, ready economic interpretation and ability to mimic some important features of Ghanaian financial series means that they deserve the attention of the Actuary seeking to model jointly the behavior of inflation, interest rates equity and other variables affecting asset liability management.

\section{Recommendations}

After further scrutiny of the models and re-estimations of the parameters, this research recommends that:

(1) The Wilkie model may be used for assessing asset allocations.

(2) The WT model may be used to determine the extreme values before using the Wilkie model for solvency calculations for both firms and regulators.

(3) The Wilkie model may be incorporated in the asset liability management programs.

(4) The Wilkie model may be incorporated into a variety of insurance applications, including dynamic financial analysis, cash flow testing, solvency testing, and operational planning.

\section{Further Research}

(1) Other time series analysis of the financial series considering the co-integration of the variables should be looked at to provide the possible relationships between the variable for further model building.

(2) Since any model cannot be accepted blindly for use no matter how better it fits the historical data, actuaries should always test the robustness of the assumptions underlying the models. Therefore further studies are necessary to check the robustness of both the Wilkie and WT models using Ghanaian data.

(3) It is recommended that future studies should be conducted on the performance of others multi-asset models for use in Ghana since this study only looked at three models.

(4) Investigation of data from other counties, especially those that have experienced high and variable inflation (sub-Saharan African) is also necessary to initiate a regional discussion on asset liability modeling.

(5) Other model selection criteria can be applied to these models using the data from Ghana especially techniques that will be able to rank the models since this research failed to rank the three models to provide a clearer judgment.

\section{References}

[1] M. Sherris, L. Tedesco and B. Zehnwirth, "Stochastic Investment Models: Unit Roots, Cointegration, State Space and GARCH Models," Actuarial Research Clearing House, vol. 1, pp. 95-144., 1997.

[2] A. Ford et. al., "Report of the maturity guarantees working party," Journal of the Institute of Actuaries, vol. 107, pp. 103212., 1980.

[3] A. D. Wilkie, "Some applications of stochastic investment models," Journal of the Institute of Actuaries Students' Society, vol. 29, pp. 25-51, 1986.

[4] R. J. Thompson, "Stochastic investment modeling: the case of South Africa," British Actuarial Journal, vol. 2, pp. 765-801, 1997.

[5] S. P. Whitten and R. G. Thomos, "A Non-linear Stochastic Model for Actuarial Use," British Actuarial Journal, vol. 5, pp. 919-953, 1999.

[6] S. Hardwick and A. Bice, "An International Survey of AssetLiability Solvency Management for Life Insurers.," AFIR, vol. 2000, 1999.

[7] W. S. Chan and S. Wang, "The Wilkie Model for Retail Price Inflation Revisited," British Actuarial Journal, vol. 4, no. 3, p. 637-652., 1998.

[8] A. D. Wilkie, "More on a Stochastic Asset Model for Actuarial Use," British Actuarial Journal, vol. 1, pp. 777-964, 1995.

[9] M. D. Ross, "Modeling a with-profits life office," British Actuarial Journal, vol. 116, pp. 691-715., 1989.

[10] I. D. Wright, "A stochastic asset model using vector autoregression; Actuarial Research Paper No. 108.," Department of Actuarial Science and Statistics, City University, London, 1998.

[11] M. Metz and M. Ort, "Stochastic models for the Swiss consumer's price index and the cost of the adjustment of pensions to inflation for a pension fund," International Colloquium, vol. 2, pp. 789-806, 1993.

[12] R. Deaves, "Modelling and predicting Canadian inflation and interest rates," Canadian Institute of Actuaries, Ontario, 1993.

[13] C. D. Daykin, T. Pentikainen and M. \& Pesonen, Practical Risk Theory for Actuaries, Chapman \& Hall., 1994.

[14] E. Frees, Y. C. Kung, M. Rosenberg, V. Young and S. W. Lai, "Forecasting social security actuarial assumptions," North American Actuarial Journal, vol. 1, pp. 49-77., 1997.

[15] R. J. Thompson, "A Stochastic Investment Model for Actuarial Use in South Africa," Transactions of the Actuarial Society of South Africa, Johanisberg, 1994.

[16] M. Sherris, L. Tedesco and B. Zehnwirth, "Investment returns and inflation models: some Australian evidence," British Actuarial Journal, vol. 5, pp. 237-268, 1999. 
[17] P. J. Lee and A. D. Wilkie, "A Comparison of Stochastic Asset Models," in Proceedings of the 10th AFIR Colloquium, Tromsoe, 2000.

[18] D. Kwiatowski, P. C. Phillips, P. Schmidt and Y. Shin, "Testing the null hypothesis of stationarity against the alternative of a unit root," Journal of Econometrics, vol. 54, p. 159-178., 1992.

[19] S. Sahin, Stochastic investment models for actuarial use in the UK, Doctor of Philosophy thesis, Department of Actuarial Mathematics and Statistics, School of Mathematical and Computer Sciences, Heriot-Watt University, 2010.

[20] F. Redington, "Review of the principles of life office valuations," Journal of the Institute of Actuaries, vol. 78, pp. 1-40., 1952.

[21] P. P. Huber, "A review of Wilkie's stochastic investment model. Actuarial Research Paper No. 70.," City University, London, 1995.

[22] G. E. P. Box and G. M. Jenkins, Time Series Analysis, Forecasting and Control, San Francisco: Holdon Day, 1976.

[23] S. Potter, "A nonlinear Approach to U.S. GNP," Journal of Applied Econometrics, vol. 10, pp. 109-125, 1995.

[24] C. Brooks, Introductory Econometrics for Finance, Cambridge University Press, 2008.

[25] P. H. Franses and D. Van Dijk, Nonlinear Time Series Models in Empirical Finance, Cambridge University Press (Virtual Publishing), 200.
[26] H. Tong, Non-linear time series: a dynamical systems approach, London: Oxford University Press, 1990.

[27] H. Tong, "On a threshold model," in Pattern Recognition and Signal Processing, Amsterdam, 1978.

[28] A. J. B. Cairns, "A multifactor equilibrium model for the term structure and inflation," in Proceedings of the 9th International AFIR Colloquium, Tokyo, 1999.

[29] Y. Yakoubov, M. Teeger and D. B. Duval, "A stochastic investment model for asset and liability management," in Proceedings of the 9th International AFIR Colloquium, Tokyo.

[30] J. Hibbert, P. Mowbray and C. Turnbull, "A Stochastic Asset Model \& Calibration for Long-Term Financial Planning Purposes, Technical Report," Barrie \& Hibbert Limited., 2001.

[31] S. Sahin, A. J. G. Cairns, T. Kleinow and A. D. Wilkie, "Revisiting the Wilkie Investment Model," in Proceedings of the 18th AFIR Coloquium, Rome, 2008.

[32] R. F. Engle, "Autoregressive conditional heteroscedasticity with estimates of the variance of United Kingdom inflation," Econometrica, vol. 50, pp. 987-1008., 1982.

[33] P. Huber, "A Review of the Wilkie's Stochastic Investment Model," British Actuarial Journal, vol. 3, no. 1, pp. 181-210., 1997.

[34] C. D. Daykin and G. B. Hey, "Managing uncertainty in a general insurance company," J. I. A., vol. 117, pp. 173-277., 1990. 